The survey was open for completion for 3 months from March 2011.

Results In total, 227 SAS doctors (78\% female, $22 \%$ male) completed the survey, $74 \%$ were on the new SAS contract, $44 \%$ as specialty doctors, $30 \%$ as associate specialists. Uptake was estimated at $40 \%$ on local assessment. According to the data in abstract P187 table 1, 1049-1253 genitourinary medicine (GUM) sessions/week are done by the respondents. Numbers are likely to be much higher given the estimated response rate. Significant numbers of HIV and sexual and reproductive health (SRH) sessions are also undertaken. Respondents indicated that $63 \%$ planned to retire within the next 15 years; $11 \%$ by $2013,18 \%$ between 2014 and 2016, 20\% between 2017 and 2022, 21\% between 2022 and 2026, 29\% were unsure when in the next 15 years they would retire.

Abstract P187 Table 1 Number of sessions performed by SAS doctors

\begin{tabular}{|c|c|}
\hline & $\begin{array}{l}\% \text { of SAS doctors } \\
\text { working these sessions }\end{array}$ \\
\hline \multicolumn{2}{|c|}{ Number of GUM sessions/week } \\
\hline $1-2$ & 20 \\
\hline $3-4$ & 20 \\
\hline $5-6$ & 17 \\
\hline $7-8$ & 15 \\
\hline $9-10$ & 18 \\
\hline$\geq 10$ & 3 \\
\hline \multicolumn{2}{|c|}{ Number of HIV sessions/week } \\
\hline None & 74 \\
\hline $1-2$ & 19 \\
\hline $3-4$ & 2 \\
\hline $5-6$ & 2 \\
\hline $7-8$ & 2 \\
\hline $9-10$ & 0.5 \\
\hline \multicolumn{2}{|c|}{ Number of SRH sessions/week } \\
\hline None & 49 \\
\hline $1-2$ & 24 \\
\hline $3-4$ & 10 \\
\hline $5-6$ & 7 \\
\hline $7-8$ & 4 \\
\hline $9-10$ & 5 \\
\hline
\end{tabular}

Conclusion SAS doctors provide a major contribution to sexual health service work and given that $63 \%$ plan to retire within the next 15 years this is a crisis in waiting. Failure to take this data into account when planning for the future may mean that the crisis will become a reality.

\section{P188 DO GENITOURINARY MEDICINE PHYSICIANS NEED TO KNOW ABOUT TROPICAL DISEASES?}

doi:10.1136/sextrans-2012-050601c.188

H Loftus, ${ }^{*}$ E Powles. Sheffield Teaching Hospitals NHS Foundation Trust, Sheffield, UK

Background Many individuals presenting to genitourinary medicine (GUM) clinics have travelled or may have been born outside the UK. A number of tropical infections can present with genitourinary symptoms.

Aims To investigate how many cases of schistosomiasis were diagnosed in a GUM clinic over a 3-year period, whether they were treated according to the European Association of Urology guidelines for the management of urogenital schistosomiasis and whether treatment led to symptomatic improvement.
Methods All the schistosoma serology requests from our clinic in 2009-2011 were obtained and identified as positive, negative or equivocal results. The results were separated into those from our HIV clinic and those from GUM. The notes for all the positive and equivocal results were reviewed.

Results 182 tests were performed on 168 different individuals. 151 tests $(83.0 \%)$ were carried out in HIV clinic, 31 tests $(17.0 \%)$ in GUM. $4(2.6 \%)$ of the tests carried out in HIV clinic were positive. 4 $(12.9 \%)$ of tests carried out in GUM were positive. All positive results were non-British born males ranging in age from 28 to 42 . All individuals with positive results had symptoms or signs that could have been attributed to urogenital schistosomiasis. Five of the 8 individuals had urine and stool sent to look for schistosome eggs, two had just urine analysed and 1 had neither. 6 individuals were referred to Infectious Diseases, two were managed in GUM. Seven of the 8 individuals were treated with praziquantel according to the guidelines. One individual declined treatment. Of those individuals treated, two had full resolution of signs and symptoms, three had partial resolution, one was followed-up in another department and one had no resolution of symptoms.

Discussion Genitourinary medicine physicians should consider a diagnosis of schistosomiasis in at-risk individuals when standard tests have not provided a diagnosis and resolution of symptoms.

\section{P189 A NATIONAL MENTORING SCHEME WITHIN GENITOURINARY MEDICINE (GUM): IS IT WORKING?}

doi:10.1136/sextrans-2012-050601c.189

${ }^{1} \mathrm{C}$ E Cohen, ${ }^{*}{ }^{2} \mathrm{E} F o x,{ }^{3} \mathrm{I}$ Fernando, ${ }^{4} \mathrm{~J}$ Dhar, ${ }^{5} \mathrm{G}$ Singh, ${ }^{6} \mathrm{H}$ Mullan, ${ }^{7} \mathrm{E}$ Street, ${ }^{8} \mathrm{G}$ Rooney. ${ }^{1}$ Chelsea \& Westminster Hospital, London, UK; ${ }^{2}$ Kent Community Health NHS Trust, Kent, UK; ${ }^{3}$ New Royal Infirmary, Edinburgh, UK; ${ }^{4}$ Leicester Royal Infirmary, Leicester, UK; ${ }^{5}$ Cobridge Community Health Centre, Staffordshire, UK; ${ }^{6}$ West Hertfordshire Hospitals NHS Trust; ${ }^{7}$ Leeds General Infirmary, Leeds, UK; ${ }^{8}$ Great Western Hospital, Swindon, UK

Background Collaboration between BASHH and the Royal College of Physicians saw the development of a National mentoring scheme for newly qualified consultants in GUM. Mentors were recruited from senior GUM clinicians, and invited on a tailored mentoring course. On appointment, new consultants are offered and allocated a mentor for 18 months.

Objectives To determine the effectiveness of the mentoring scheme thus far

Methods Voluntary interim questionnaires were distributed via Survey Monkey to mentor/mentee pairs who had joined the scheme for $>3$ months in January 2011. Responses were anonymous and quantitative data are presented.

Results 18 mentees and 17 mentors responded. The mean time from mentor allocation was 9.1 months (ranging $3-17$ ). $80 \%$ of mentees found it easy to arrange their first meeting with their mentor, $72 \%$ had met their mentor between 1 and 4 times in person. Almost three-fourth $(71 \%)$ felt they had received ample contact with their mentor, and in those who hadn't, time constraints and multiple competing service demands were repeatedly cited as barriers Encouragingly, 69\% of mentees felt the programme had helped them, with a further $25 \%$ responding, "not yet" as it was "too early in their mentorship". 93\% of mentors responded they felt confident to support their mentee, and $79 \%$ perceived the relationship with their mentee was going well. Mentee feedback particularly favoured greater structure, including alerts to encourage meeting prioritisation and further guidance on what could be covered within mentorship.

Discussion The mentoring scheme, which now hosts $67 \mathrm{BASHH}$ mentors and 41 mentees, is providing significant support to new GUM consultants. By developing a mentoring module with clear 